\title{
Avoid Vendor Lock-In using Virtual Adaptive Framework (VAF)
}

\author{
Ch. Siva Rama Krishna \\ M.Tech(CSE) \\ KHIT,Guntur, \\ India
}

\author{
B. Tarakeswara Rao, PhD \\ Professor(Dept.of CSE) \\ KHIT, Guntur, \\ India
}

\author{
B. Sathyanarayana \\ Reddy \\ Associate Professor(Dept.of \\ CSE) \\ KHIT, Guntur \\ India
}

\begin{abstract}
The greater part of the present cloud computing platforms offer Infrastructure as a Service (IaaS) model, which expects to procurement fundamental virtualized computing assets as on interest and element Services. By the by, a single cloud does not have boundless assets to offer to its clients, consequently the thought of an Inter-Cloud environment where a cloud can utilize the infrastructure assets of different clouds. Because of vendor lock-in snags in broad daylight cloud computing the capacity to define transferable cloudbased Services is pivotal yet has not yet been comprehended palatably. . The genuine cutting edge cloud services configuration does not methodically manage how to define, send and work cross-platform competent cloud services. This is mainly because of the inherent multifaceted nature of the field and contrasts in subtle elements between a lot of existing open and private cloud infrastructures. In any case, there is no basic structure in presence that permits the service proprietors to consistently procurement even some essential services across different cloud Service Providers, to address the above issues I recommend Virtualized Private cloud with versatile system, which drives the displayed issues, thus keeping in mind the end goal to give the boundless services, it will demonstrate high security, data accessibility, data integrity with elite.
\end{abstract}

\section{Keywords}

Cloud Computing, Vendor Lock-In, Cross-Platform, Interclub Virtual adaptive framework

\section{INTRODUCTION}

Cloud computing can be seen as another IT Service conveyance worldview frequently called a "programmable data centre" .These networks open "programmable data centers" give a few interesting elements. They permit scaling here and there asset utilization on an as-required premise prone to give expense preferences (particularly in crest burden situations [2] [3]) because of their prudent pay-as-you-go standard and specialized brisk scaling abilities. Financially they transform settled expenses into variable expenses making subject particularly interesting for inventive new businesses not ready to do noteworthy in advance speculations. While new businesses, as a rule, don't have an entrenched plan of action, little and medium-sized undertakings have. In this way little and medium-sized undertakings are regularly imaginative from one perspective additionally exceptionally watchful in ascertaining dangers then again. Adjacent to changes these organizations are likewise centred on staying away from barely measurable dangers. So adjacent to the aforementioned points of interest, normal security, Service, accessibility, expense concerns and particularly innovative seller lock-in stresses frequently join cloud computing which is particularly valid for little and medium-sized endeavours. While security and Service concerns frequently can be replied by encryption [2], and expense concerns can be replied by expense based choice making models [2] [3], seller lock-in issues sit tight. From the mechanical perspective genuine best in class cloud-based programming, Services are regularly described by a very verifiable innovative reliance on basic facilitating cloud bases. This article concentrates on this seller lock-in an issue and determines a few viewpoints to be explained to overcome merchant lock-in issues improving cloud computing a fit for new companies, for little, medium and huge estimated endeavours also. Seller lock-in is characterized to make a client reliant on a merchant for items and Services, not able to utilize another merchant without significant exchanging expenses. This is precisely the real best in a class of IaaS (base as an Service) cloud computing. On one hand IaaS gives a product Service. Merchandise depicts a class of requested products (for a situation of IaaS computing, stockpiling and systems Service Services) without subjective separation. So the business sector regards examples of the merchandise as (almost) proportionate. On the other hand more straightforward: a virtual server gave by Google Compute Engine (GCE) is not superior to a server gave by Amazon Web Services (AWS). Both can be utilized to have a client particular Service. So IaaS gives wares from a basic perspective. Then again, reality looks more perplexing. Despite the fact that a considerable measure of cloud computing Services are aware benefits, a change starting with one Service Providers then onto the next Service Providers is by and large not as simple as purchasing espresso from an alternate Providers. This is essential because of characteristic conditions on hidden cloud frameworks. These conditions are frequently subliminal created by cloud Service Providers' particular (non-institutionalized) Service APIs. To overcome subliminally created merchant lock-ins, this paper proposes an idea called lightweight virtualization group (LVC) depending on working framework virtualization. Lightweight (or working framework) virtualization is increasing more consideration in cloud computing. The vast majority of the at present accessible Cloud Computing arrangements are for the most part centered around giving functionalities and Services at the framework level, e.g., enhanced execution for virtualization of computing, stockpiling and system assets, and also essential central usefulness, for example, virtual machine (VM) movements and server solidification and so on. In the situations when the more elevated amount and more conceptual concerns should be tended to, existing Infrastructure as a Service (IaaS) arrangements tend to concentrate on useful perspectives just. Moreover, if a cloud's 
computational and stockpiling foundation assets are overburden because of expanded workloads, its Service towards it customers will debate.

The thought of an Inter-Cloud [1] has been increasing much footing to address such a circumstance, where a cloud can get the required framework assets of different clouds. On the other hand, keeping in mind the end goal to advance from an essential cloud Service framework to a more versatile cloud Service biological system, there is an incredible requirement for instruments and Services that bolster and give more elevated amount concerns and non-useful angles in a thorough way. The OPTIMIS venture [2] is a continuous exertion in such manner which endeavours to give a comprehensive way to deal with cloud Service offering so as to provision a solitary reflection for numerous existing together cloud architectures. Of the different abnormal state concerns being tended to by the OPTIMIS venture, a noteworthy worry of high significance is the provisioning of a secure communication framework to the Services using the assets of distinctive cloud IaaS Providers. The utilization example of these Services is normally entirely adaptable, i.e., on one hand they may be specifically gotten to by end-clients or, then again, they may be coordinated by other Service Providers (SP) for their clients. There are three key strides in the life cycle of an Service in the cloud computing environment; the development of the Service, the arrangement of the support of one or more IaaS clouds and, in conclusion, the operational Service of the Service. In the subsequent situations, the vicinity of the various IaaS Providers in the cloud environment is the key issue that should be tended to by any inter-cloud security arrangement. A noteworthy objective of Service proprietors is to choose IaaS Providers in a proficient route keeping in mind the end goal to have the distinctive parts of their Services on fitting clouds. In this appreciation, outsider cloud intermediaries [3] can assume a noteworthy part in disentangling the utilization, execution, and conveyance of the cloud Services. These intermediaries can likewise offer an intermediation layer crossing over numerous cloud Providers to convey a large group of streamlining and esteem included Services which exploit the horde individual cloud Services e.g., accumulation of distinctive Services or assertion for a best-match Service from various comparable Services. For the various interaction conceivable outcomes among these gatherings, whatever the utilization situations perhaps, the security of data and the communication between the purchasers of the Service and its different Providers is of fundamental significance. In the light of the above examination, it is clear that an inter-cloud security arrangement is exceedingly alluring that would give a framework empowering consistent and secure communication between the performing artists of a cloud biological system over various cloud stages. Such an answer, be that as it may, needs to beat various difficulties due to building restrictions. This is on account of a large portion of the present cloud Service stages, and the multi-occupants situations they offer, make it hard to give the buyers of their Services adaptable and versatile control over the centre security parts of their Services like encryption, communication segregation, and key Service. Secure communication is likewise tested by the absence of element system reconfigurability in most cloud Providers, created by the characteristic impediments of the settled system architectures offered by these Providers. In this work we address the secure, adaptable and versatile communication worries that in our perspective must be overcome keeping in mind the end goal to give comprehensive provisioning of Services to buyers from numerous cloud Service Providers.
We display the construction modelling and plan of an intercloud secure communication framework that offers the elements of dynamic and adaptable virtual system development, productive and versatile key Service and negligible manual arrangement all on top of secure and private communication between the parts of the Service over different cloud stages.

\section{VENDOR LOCK-IN OVER COMPUTING \\ 2.1.How Vendor Lock-In emerges technically in Cloud Computing}

It is anything but difficult to make cloud looking so as to compute seller lock-in issues clear at an illustration. Investigating gave $\mathrm{s}$ by Amazon Web Services we see that AWS gives more than 30 benefits yet just nine of them can be evaluated as were. Utilizing cloud computing means it is likely that (flat) versatile and flexible frameworks will be sent. That is the reason cloud computing is utilized as a part of most cases. So, as a rule, versatile conveyed frameworks will be sent to cloud bases. Segments of dispersed frameworks need to interact and this can be dubious in points of interest for framework engineers. While the centre parts of these appropriated frameworks can be sent utilizing thing Services every single further Service to coordinate these virtualized server cases in a flexible and adaptable way are given by nonware Services. If there should arise an occurrence of AWS it is likely that Services like versatile burden adjusting, auto scaling, message line framework are expected to plan a flexible and adaptable framework on an AWS foundation. Be that as it may, particularly these Services are non-merchandise Services tying an organization to seller particular framework .The same is valid for other cloud Service Providers .All cloud Service Providers attempt to animate cloud clients to utilize non-item accommodation Services to tough situation them to their framework. From a cloud Service Providers perspective, the wish to tie clients is not surprising thus the specified procedure is more than self-evident. By and by from a cloud client perspective this seller lock-in ought to be maintained a strategic distance from to stay adaptable in operation Reflecting genuine examination writing the accompanying systems can be distinguished to overcome merchant lock-in

\subsection{Modern top-down institutionalization methodologies like CIMI}

Attempt to characterize an institutionalized Programming interface to diverse cloud Service Providers. So on the off chance that it is conceivable to send to Providers A by means of an institutionalized Programming interface it is likewise conceivable to convey to a (standard consistent) Providers B. Taking a gander at CIMI [6] in point of interest will demonstrate that average institutionalization issues emerge like the minimum shared factor issue. Institutionalization appears to be just to work for where Services however not for non item benefits vital for arrangements of regular many-sided quality (burden adjusted, flexible and dispersed). Besides organizations like AWS are not known not extremely standard situated.

\subsection{Open stack bottom up methodologies}

Attempt to do likewise like the modern institutionalization approach yet applying a base up a system is a Ruby-based Programming interface to access more than 30 cloud Service Providers. Be that as it may, as CIMI fog.io must utilize a slightest shared factor approach. More mind boggling Services like burden adjusting, auto scaling, informing, and so on are 
not open (or in the event that, they are indeed Providers particular). A primary issue of the praiseworthy fog.io library is it's extremely Providers particular parameter sets. Organizations like Netflix or PayPal create open source making more mind boggling Services for burden adjusting, auto scaling, and so on available for private cloud foundations like Eucalyptus3 or OpenStack4. Be that as it may, an exchange between these universes is not really conceivable at this time in light of the fact that they appear to depend on a great deal of exceptionally base particular elements.

\subsection{More formal examination methodologies}

Attempt to formalize the arrangement depiction by characterizing generally XMLbased sending dialects .Utilizing a conveying part skilled to interpret the arrangement dialect proposals methodologies are proficient in sending these formal depictions to the APIs of a few Service Providers (even into unified clouds). In any case, these conveyed frameworks are not versatile (so don't give programmed all over scaling). Besides they don't confront the issue how to move a conveyed appropriated framework starting with one Service Providers then onto the next. So far the majority of the systems appear to have weaknesses particularly in taking care of element cloud organizations of regular intricacy (so arrangements which are versatile and burden adjust

\subsection{Service Level Agreement}

Service-level agreement (SLA) is a contract between a network service provider and a customer that specifies, usually in measurable terms (QoS), what services the network service provider will furnish

- Performance guarantee metrics

- Up-time and down-time ratio ,System throughput and Response time

- Problem management detail ,Penalties for non-performance and Documented security capabilities

\subsection{Avoid Vendor Lock-In Using Virtual adaptive}

framework (VAF)

IaaS Cloud computing is intensely influenced by hypervisor virtualization. Various virtual servers giving distinctive working frameworks can be keeping running on one physical host framework. So clients can run the working framework they need. In this paper, we exhibit the outline and construction modelling of such a Virtual adaptive framework (VAF) arrangement, which gives secure correspondence offices to clients that need to convey their cloud administration's segments over the foundation of Metacloud cloud IaaS Providers. At its centre, it gives the capacity to consequently set up P2P overlay systems among the VMs constituting a solitary cloud benefit that keeps running on various IaaS Providers. We handle the stage interoperability and league issues inalienable in the between cloud environment by utilizing a VM contextualization way to deal with procurement our virtual machines on various cloud stages as a piece of our VAF arrangement's operation. Utilizing the same $\mathrm{P} 2 \mathrm{P}$ and $\mathrm{VM}$ contextualization strategies, we likewise offer a conveyed key administration which encourages the dissemination of cryptographic builds like keys and authentications to the VMs constituting the client's cloud administration. The design and support of the VPN associations utilizing the $\mathrm{P} 2 \mathrm{P}$ overlay are self-governing and straightforward to the cloud administration itself, as a noteworthy objective of our work is to free the cloud administration client from the convoluted setups normally required to set up the key administration and virtual systems administration foundations in comparative issue spaces.

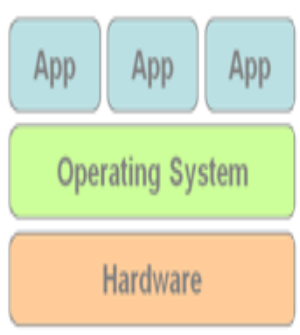

Traditional Stack

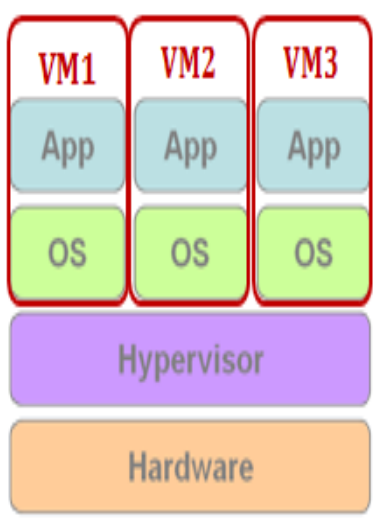

Virtualized Stack
Fig 1. Virtual adaptive framework

- Virtualization technique shift OS onto hypervisor.

- Multiple OS share the physical hardware and provide different services.

- Improve utilization, availability, security and convenience.

\subsection{Multi-tenant Design}

Multi-occupant alludes to a guideline in software architecture where a solitary case of the software keeps running on a server, serving numerous customer associations. With a multiinhabitant architecture, a software application is intended to for all intents and purposes parcel its information and design along these lines every customer association works with a tweaked virtual application case

\subsection{Requirements of a Lightweight Virtualization Cluster}

Client oriented requirements:

a. Customization

i. Multi-tenant applications are typically required to provide a high degree of customization to support each target organization's needs.

b. Quality of service

i. Multi-tenant applications are expected to provide adequate levels of security and robustness.

\section{IMPORTANT SECURITY AND PRIVACY ISSUES \\ a. Data Protection}

ii. To be viewed as secured, information from one client must be appropriately isolated from that of another. 


\section{b. Identity Management}

iii. Every undertaking will have its own character administration framework to control access to data and registering assets.

\section{c. Application Security}

iv. Cloud Providers ought to guarantee that applications accessible as an administration by means of the cloud are secure.

\section{d. Privacy}

v. Providers guarantee that all basic information are conceal and that just approved clients have entry to information completely

\section{COLLABORATION WITH CLOUD PROVIDERS WITH ADVANTAGES}

IT resources are centrically managed and optimized

i. Cloud Providers fabricates execution advanced equipment

ii. Cloud Providers constructs united cooling framework

iii. Cloud Providers will consider the geographic issues

iv. Cloud Providers will consider legitimate arrangement issues

\section{Some benefits}

a. IT infrastructure can be shared among enterprises

b. IT infrastructure performance and utilization can be optimized

c. Large-scale integrated optimization can be applied
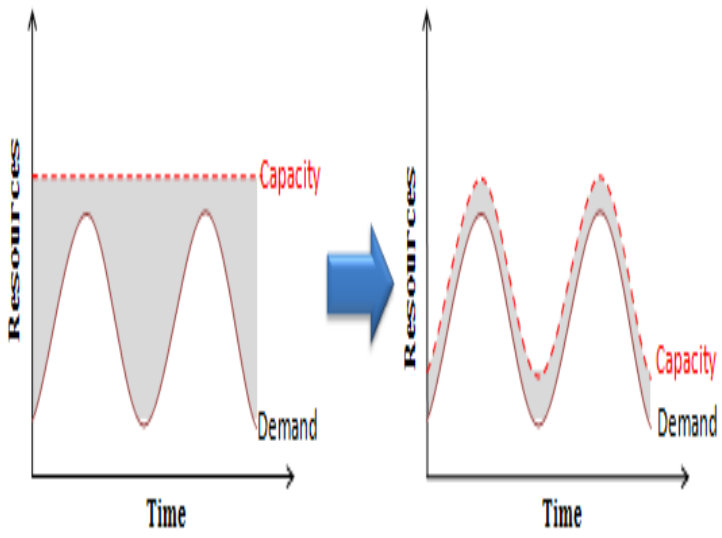

Fig 2. Dynamic provisioning Cloud resources

Dynamic provisioning Cloud resources should be provisioned dynamically

- Meet seasonal demand variations

- Meet demand variations between different industries

- Meet burst demand for some extraordinary events

\section{CONCLUSIONS}

In this paper we suggested Virtual adaptive framework (VAF) which leads Meta cloud issues and it avoids vendor-lock in and provide Meta cloud quality of services with data integrity and data availability it provides many benefits like infrastructure can be shared among enterprises, IT infrastructure performance and utilization can be optimized and Large-scale integrated optimization can be applied

\section{REFERENCES}

[1] A. Rowstron, P. Druschel, Pastry: Scalable, decentralized object location,and routing for Large-Scale Peer-to-Peer systems, in: Middleware 2001, 2001.

[2] B. Y. Zhao, L. Huang, J. Stribling, S. C. Rhea, A. D. Joseph, J. D. Kubiatowicz, Tapestry: a resilient globalscale overlay for service deployment, Selected Areas in Communications, IEEE Journal on

[3] P. "Maymounkov, D. Mazi'eres, "kademlia: A peer-topeer information system based on the xor metric", in: "Revised Papers from the First International Workshop on Peer-to-Peer Systems", "Springer-Verlag","2002".

[4] J. Douceur, The sybil attack, in: Peer-to-Peer Systems, Springer Berlin / Heidelberg, 2002.

[5] D. Taylor, T. Wu, N. Mavrogiannopoulos, T. Perrin, Using the Secure Remote Password (SRP) Protocol for TLS Authentication, RFC 5054, 2007.

[6] A. S. Dan Boneh, B. Waters, Functional encryption: a new vision for public-key cryptography, Commun. ACM 55 (11) (2012) 56-64.

[7] Barr, J. (2010) Host Your Web Site in the Cloud: Amazon Web Services Mad Easy. Sitepoint.

[8] Kratzke, N. (2012) Cloud Computing Costs and Benefits-An IT Management Point of View. In: Ivanov, I., van Sinderen,M. and Shiskov, B., Eds., Cloud Computing and Services Sciences, Springer, New York, 185-203.

[9] Kratzke, N. (2011) Overcoming Ex Ante Cost Intransparency of Clouds-Using System Analogies and a Corresponding Cost Estimation Model. CLOSER 2011-1st International Conference on Cloud Computing and Services Science (Special Session on Business Systems and Aligned IT Services-BITS 2011) Noordwijkerhout, 7-9 May 2011, 707-716.

[10] Satzger, B., Hummer, W., Inzinger, C., Leitner, P. and Dustdar, S. (2013) Winds of Change: From Vendor Lock-In to the Meta Cloud. Internet Computing, IEEE, $17,69-73$

[11] Amazon Web Services (2014) Whitepaper "Overview of Amazon Web Services".https://s3.amazonaws.com/awsmedia/AWS_O verview.pdf

[12] DMTF, CIMI (Cloud Infrastructure Management Interface) (2014) Last Access 30th June 2014.http://dmtf.org/standards/cloud

[13] Fog.io (The Ruby Cloud Services Library) (2014) Last Access 30th June 2014. http://fog.io

[14] de Alfonso, C., Caballer, M., Alvarruiz, F., Molto, G. and Hernandez, V. (2011) Infrastructure Deployment over the Cloud. Proceedings of 2011 IEEE 3rd 
International Journal of Computer Applications (0975 - 8887)

Volume 133 - No.1, January 2016

International Conference on Cloud Computing Technology and Science (Cloud-Com), Athens, 29 November-1 December 2011, 517-521.

[15] Juve, G. and Deelman, E. (2011) Automating Application Deployment in Infrastructure Clouds. Proceedings of 2011 IEEE 3rd International Conference on Cloud Computing Technology and Science
(CloudCom), Athens, 29 November-1 December 2011, 658-665.

[16] Lenk, A., Danschel, C., Klems, M., Bermbach, D. and Kurze, T. (2011) Requirements for an IaaS Deployment Language in Federated Clouds. Proceedings of SOCA, Irvine, 12-14 December 2011, 1-4. 